\title{
Existence and Ulam stability results for nonlinear hybrid implicit Caputo fractional differential equations
}

\author{
Adel Lachouri, Abdelouaheb Ardjouni, Ahcene Djoudi
}

\begin{abstract}
In this paper, we study the existence, uniqueness and estimate of solutions for nonlinear hybrid implicit Caputo fractional differential equations by using the contraction mapping principle and the generalization of Gronwall's inequality. After that, we also establish the Ulam stability for the problem at hand. Finally, an example is given to illustrate this work.
\end{abstract}

\section{INTRODUCTION}

The concept of fractional calculus is a generalization of the ordinary differentiation and integration to arbitrary non integer order. Fractional differential equations with and without delay arise from a variety of applications including in various fields of science and engineering such as applied sciences, practical problems concerning mechanics, the engineering technique fields, economy, control systems, physics, chemistry, biology, medicine, atomic energy, information theory, harmonic oscillator, nonlinear oscillations, conservative systems, stability and instability of geodesic on Riemannian manifolds, dynamics in Hamiltonian systems, etc. In particular, problems concerning qualitative analysis of linear and nonlinear fractional differential equations with and without delay have received the attention of many authors, see [1]-[28], [30]-[36], [38]-[40] and the references therein. The study of Ulam stability for fractional differential equations was initiated by Wang et al. [39]. An overview on the development of theory of the Ulam-Hyers and the Ulam-Hyers-Rassias stability for fractional differential equations can be found in $[39,40]$ and the references given therein. Subsequently, many authors discussed various Ulam-Hyers stability problem for different kinds of fractional integral and fractional differential equations by using different techniques, see $[10,14,32,39,40]$ and the references therein.

2010 Mathematics Subject Classification. Primary: 34A12; Secondary: 34K20, 45N05.

Key words and phrases. Implicit fractional differential equations, Caputo fractional derivatives, fixed point theorems, existence, uniqueness, Ulam stability.

Full paper. Received 24 December 2019, revised 9 April 2020, accepted 10 April 2020, available online 4 May 2020. 
Hybrid differential equations arise from a variety of different areas of applied mathematics and physics, e.g., in the deflection of a curved beam having a constant or varying cross section, a three-layer beam, electromagnetic waves or gravity driven flows and so on [4, 20, 22, 21, 38].

Dhage and Lakshmikantham [21] discussed the existence of solutions for the following first-order hybrid differential equation

$$
\left\{\begin{array}{l}
\frac{d}{d t}\left(\frac{x(t)}{g(t, x(t))}\right)=f(t, x(t)) \text { a.e. } t \in\left[t_{0}, t_{0}+T\right], \\
x\left(t_{0}\right)=x_{0}, x_{0} \in \mathbb{R},
\end{array}\right.
$$

where $t_{0}, T \in \mathbb{R}$ with $T>0, g:\left[t_{0}, t_{0}+T\right] \times \mathbb{R} \rightarrow \mathbb{R} \backslash\{0\}$ and $f:\left[t_{0}, t_{0}+T\right] \times$ $\mathbb{R} \rightarrow \mathbb{R}$ are continuous functions. By using the fixed point theorem in Banach algebra, the authors obtained the existence results.

The hybrid fractional differential equation

$$
\left\{\begin{array}{l}
D^{\alpha}\left(\frac{x(t)}{g(t, x(t))}\right)=f(t, x(t)) \text { a.e. } t \in[0, T], \\
x(0)=0
\end{array}\right.
$$

has been investigated in [38], where $D^{\alpha}$ is the Riemann-Liouville fractional derivative of order $0<\alpha<1, g:[0, T] \times \mathbb{R} \rightarrow \mathbb{R} \backslash\{0\}$ and $f:[0, T] \times \mathbb{R} \rightarrow \mathbb{R}$ are continuous functions. By employing the fixed point theorem in Banach algebra, the authors obtained the existence of a solution.

In [14], Benchohra and Lazreg established four types of Ulam stability for the following implicit fractional differential equation

$$
\left\{\begin{array}{l}
{ }^{C} D^{\alpha} x(t)=f\left(t, x(t),{ }^{C} D^{\alpha} x(t)\right), t \in[0, T], 0<\alpha \leq 1, \\
x(0)=x_{0}, x_{0} \in \mathbb{R},
\end{array}\right.
$$

where ${ }^{C} D^{\alpha}$ is the Caputo fractional derivative, $f:[0, T] \times \mathbb{R} \times \mathbb{R} \rightarrow \mathbb{R}$ is a given continuous function.

In [10], Ardjouni and Djoudi studied the existence, interval of existence and uniqueness of solutions for the hybrid implicit Caputo fractional differential equation

$$
\left\{\begin{array}{l}
{ }^{C} D^{\alpha}\left(\frac{x(t)}{g(t, x(t))}\right)=f\left(t, x(t),{ }^{C} D^{\alpha}\left(\frac{x(t)}{g(t, x(t))}\right)\right), t \in[0, T], \\
x(0)=\eta g(0, x(0)), \eta \in \mathbb{R},
\end{array}\right.
$$

where $f:[0, T] \times \mathbb{R} \times \mathbb{R} \rightarrow \mathbb{R}$ and $g:[0, T] \times \mathbb{R} \rightarrow \mathbb{R} \backslash\{0\}$ are nonlinear continuous functions and ${ }^{C} D^{\alpha}$ denotes the Caputo fractional derivative of order $0<\alpha<1$.

Motivated by the above works, we study the existence and uniqueness of solution and the Ulam stability for the following nonlinear hybrid implicit Caputo fractional differential equation

$$
\left\{\begin{array}{l}
{ }^{C} D^{\alpha}\left(\frac{x(t)-f(t, x(t))}{g(t, x(t))}\right)=h\left(t, x(t),{ }^{C} D^{\alpha}\left(\frac{x(t)-f(t, x(t))}{g(t, x(t))}\right)\right), t \in[0, T] \\
x(0)=\theta g(0, x(0))+f(0, x(0)), \theta \in \mathbb{R}
\end{array}\right.
$$


where $f:[0, T] \times \mathbb{R} \rightarrow \mathbb{R}, g:[0, T] \times \mathbb{R} \rightarrow \mathbb{R} \backslash\{0\}$ and $h:[0, T] \times \mathbb{R} \times \mathbb{R} \rightarrow \mathbb{R}$ are nonlinear continuous functions and ${ }^{C} D^{\alpha}$ denotes the Caputo fractional derivative of order $0<\alpha<1$. To show the existence, uniqueness and estimate of solutions of (1), we transform (1) into an integral equation and then use the contraction mapping principle and the generalization of Gronwall's inequality. Further, we obtain Ulam-Hyers and Ulam-Hyers-Rassias stability results of (1). Finally, we provide an example to illustrate our obtained results.

The rest of this paper is organized as follows. Some definitions from fractional calculus theory are recalled in Section 2. In Section 3, we will prove the existence, uniqueness and estimate of solutions for (1). In Section 4, we discuss the Ulam stability results. Finally, an example is given in Section 5 to illustrate the usefulness of our main results.

\section{Preliminaries}

In this section we present some basic definitions, notations and results of fractional calculus which are used throughout this paper.

Definition 1 ([30]). The fractional integral of order $\alpha>0$ of a function $x: \mathbb{R}^{+} \rightarrow \mathbb{R}$ is given by

$$
I^{\alpha} x(t)=\int_{0}^{t} \frac{(t-s)^{\alpha-1}}{\Gamma(\alpha)} x(s) \mathrm{d} s
$$

provided the right side is pointwise defined on $\mathbb{R}^{+}$.

Definition 2 ([30]). The Caputo fractional derivative of order $\alpha>0$ of a function $x: \mathbb{R}^{+} \rightarrow \mathbb{R}$ is given by

$$
{ }^{C} D^{\alpha} x(t)=I^{n-\alpha} D^{(n)} x(t)=\int_{0}^{t} \frac{(t-s)^{n-\alpha-1}}{\Gamma(n-\alpha)} x^{(n)}(s) \mathrm{d} s,
$$

where $n=[\alpha]+1$, provided the right side is pointwise defined on $\mathbb{R}^{+}$.

Lemma 1 ([30]). Let $\alpha>0$. Suppose $x \in C^{n-1}([0,+\infty), \mathbb{R})$ and $x^{(n)}$ exists almost every-where on any bounded interval of $\mathbb{R}^{+}$. Then

$$
\left(I^{\alpha C} D^{\alpha} x\right)(t)=x(t)-\sum_{k=0}^{n-1} \frac{x^{(k)}(0)}{k !} t^{k} .
$$

In particular, when $0<\alpha<1,\left(I^{\alpha C} D^{\alpha} x\right)(t)=x(t)-x(0)$.

Lemma 2 ([30]). For all $\alpha, \beta \in[0, \infty)$ and $\mu>-1$, then

$$
\int_{0}^{t}(t-s)^{\beta-1} s^{\alpha-1} \mathrm{~d} s=\frac{\Gamma(\alpha) \Gamma(\beta)}{\Gamma(\alpha+\beta)} t^{\alpha+\beta-1},
$$

and

$$
I^{\alpha} t^{\mu}=\frac{\Gamma(\mu+1)}{\Gamma(\mu+\alpha+1)} t^{\mu+\alpha} .
$$


The following generalization of Gronwall's lemma for singular kernels plays an important role in obtaining our main results.

Lemma 3 ([29]). Let $x:[0, T] \rightarrow[0, \infty)$ be a real function and $w$ is a nonnegative locally integrable function on $[0, T]$. Assume that there is a constant $a>0$ such that for $0<\alpha<1$

$$
x(t) \leq w(t)+a \int_{0}^{t}(t-s)^{-\alpha} x(s) \mathrm{d} s,
$$

then, there exists a constant $k=k(\alpha)$ such that

$$
x(t) \leq w(t)+k a \int_{0}^{t}(t-s)^{-\alpha} w(s) \mathrm{d} s .
$$

To define Ulam's stability, we consider the following fractional differential equation

$$
{ }^{C} D^{\alpha} u(t)=f(t, u(t)), 0<\alpha \leq 1, t \in[0, T] .
$$

Definition 3 ([36]). The equation (3) is said to be Ulam-Hyers stable if there exists a real number $k>0$ such that for each $\epsilon>0$ and for each $y \in C([0, T], \mathbb{R})$ solution of the inequality

$$
\left|{ }^{C} D^{\alpha} y(t)-f(t, y(t))\right| \leq \epsilon, t \in[0, T],
$$

there exists a solution $u$ of the equation (3) with

$$
|y(t)-u(t)| \leq k \epsilon, t \in[0, T] .
$$

Definition 4 ([36]). The equation (3) is said to be Ulam-Hyers-Rassias stable with respect to $\phi \in C\left([0, T], \mathbb{R}^{+}\right)$if there exists a real number $k_{\phi, f}>0$ such that for each $\epsilon>0$ and for each $y \in C([0, T], \mathbb{R})$ solution of the inequality

$$
\left|{ }^{C} D^{\alpha} y(t)-f(t, y(t))\right| \leq \epsilon \phi(t), t \in[0, T],
$$

there exists a solution $u \in C([0, T], \mathbb{R})$ of the equation (3) with

$$
|y(t)-u(t)| \leq k_{\phi, f} \phi(t) \epsilon, t \in[0, T] .
$$

Remark 1 ([36]). If there is a function $\Psi \in C([0, T], \mathbb{R})$ (dependent on $y$ ), such that

1) $|\Psi(t)| \leq \epsilon$, for all $t \in[0, T]$,

2) ${ }^{c} D^{\alpha} y(t)=f(t, y(t))+\Psi(t), t \in[0, T]$.

Then a function $y \in C([0, T], \mathbb{R})$ is a solution of the inequality (4).

Theorem 1 (Banach's fixed point theorem [37]). Let $\Omega$ be a non-empty closed convex subset of a Banach space $(S,\|\|$.$) , then any contraction map-$ ping $P$ of $\Omega$ into itself has a unique fixed point. 


\section{Existence And Uniqueness}

In this section, we give the equivalence of the initial value problem (1) and prove the existence, uniqueness and estimate of solution of (1).

The proof of the following lemma is close to the proof of Lemma 6.2 given in [19].

Lemma 4. If the functions $f:[0, T] \times \mathbb{R} \rightarrow \mathbb{R}, g:[0, T] \times \mathbb{R} \rightarrow \mathbb{R} \backslash\{0\}$ and $h:[0, T] \times \mathbb{R} \times \mathbb{R} \rightarrow \mathbb{R}$ are continuous, then the initial value problem (1) is equivalent to the nonlinear fractional Volterra integro-differential equation

$$
\begin{aligned}
x(t)= & f(t, x(t))+\theta g(t, x(t)) \\
& +\frac{g(t, x(t))}{\Gamma(\alpha)} \int_{0}^{t}(t-s)^{\alpha-1} h\left(s, x(s),{ }^{C} D^{\alpha}\left(\frac{x(s)-f(s, x(s))}{g(s, x(s))}\right)\right) \mathrm{d} s,
\end{aligned}
$$

for $t \in[0, T]$.

Theorem 2. Let $T>0$. Assume that the continuous functions $f:[0, T] \times$ $\mathbb{R} \rightarrow \mathbb{R}, g:[0, T] \times \mathbb{R} \rightarrow \mathbb{R} \backslash\{0\}$ and $h:[0, T] \times \mathbb{R} \times \mathbb{R} \rightarrow \mathbb{R}$ satisfy the following conditions:

(H1) There exists $M_{g} \in \mathbb{R}^{+}$such that

$$
|g(t, u)| \leq M_{g},
$$

for all $u \in \mathbb{R}$ and $t \in[0, T]$.

(H2) There exists $M_{h} \in \mathbb{R}^{+}$such that

$$
|h(t, u, v)| \leq M_{h},
$$

for all $u, v \in \mathbb{R}$ and $t \in[0, T]$.

(H3) There exist $K_{1}, K_{2}, K_{3} \in \mathbb{R}^{+}, K_{4} \in(0,1)$ with $K_{1}+K_{2}|\theta| \in(0,1)$ such that

$$
\begin{aligned}
\left|f(t, u)-f\left(t, u^{*}\right)\right| & \leq K_{1}|u-v|, \\
\left|g(t, u)-g\left(t, u^{*}\right)\right| & \leq K_{2}|u-v|,
\end{aligned}
$$

and

$$
\left|h(t, u, v)-h\left(t, u^{*}, v^{*}\right)\right| \leq K_{3}\left|u-u^{*}\right|+K_{4}\left|v-v^{*}\right|,
$$

for all $u, v, u^{*}, v^{*} \in \mathbb{R}$ and $t \in[0, T]$.

If

$$
\beta=K_{1}+K_{2}|\theta|+M_{h} K_{2}+\frac{M_{g} K_{3}}{\left(1-K_{4}\right)} \frac{T^{\alpha}}{\Gamma(\alpha+1)}<1 .
$$

Then, the problem (1) has a unique solution $x \in C([0, T], \mathbb{R})$.

Proof. Let

$$
{ }^{C} D^{\alpha}\left(\frac{x(t)-f(t, x(t))}{g(t, x(t))}\right)=z_{x}(t), x(0)=\theta g(0, x(0))+f(0, x(0)),
$$


then by Lemma 4, we have

$$
x(t)=f(t, x(t))+\theta g(t, x(t))+\frac{g(t, x(t))}{\Gamma(\alpha)} \int_{0}^{t}(t-s)^{\alpha-1} z_{x}(s) \mathrm{d} s,
$$

where

$$
z_{x}(t)=h\left(t, f(t, x(t))+\theta g(t, x(t))+g(t, x(t)) I^{\alpha} z_{x}(t), z_{x}(t)\right) .
$$

That is $x(t)=f(t, x(t))+\theta g(t, x(t))+g(t, x(t)) I^{\alpha} z_{x}(t)$. Define the mapping $P: C([0, T], \mathbb{R}) \rightarrow C([0, T], \mathbb{R})$ as follows

$$
(P x)(t)=f(t, x(t))+\theta g(t, x(t))+\frac{g(t, x(t))}{\Gamma(\alpha)} \int_{0}^{t}(t-s)^{\alpha-1} z_{x}(s) \mathrm{d} s .
$$

It is clear that the fixed points of $P$ are solutions of (1). Let $x, y \in$ $C([0, T], \mathbb{R})$, then we have

$$
\begin{aligned}
\mid & (P x)(t)-(P y)(t) \mid \\
= & \mid f(t, x(t))+\theta g(t, x(t))+\frac{g(t, x(t))}{\Gamma(\alpha)} \int_{0}^{t}(t-s)^{\alpha-1} z_{x}(s) \mathrm{d} s \\
& -f(t, y(t))-\theta g(t, y(t))-\frac{g(t, y(t))}{\Gamma(\alpha)} \int_{0}^{t}(t-s)^{\alpha-1} z_{y}(s) \mathrm{d} s \mid \\
\leq & |f(t, x(t))-f(t, y(t))|+|\theta||g(t, x(t))-g(t, y(t))| \\
& +|g(t, x(t))-g(t, y(t))| \frac{1}{\Gamma(\alpha)} \int_{0}^{t}(t-s)^{\alpha-1}\left|z_{x}(s)\right| \mathrm{d} s \\
& +|g(t, y(t))| \frac{1}{\Gamma(\alpha)} \int_{0}^{t}(t-s)^{\alpha-1}\left|z_{x}(s)-z_{y}(s)\right| \mathrm{d} s \\
\leq & K_{1}|x(t)-y(t)|+K_{2}|\theta||x(t)-y(t)| \\
& +K_{2}|x(t)-y(t)| \frac{M_{h}}{\Gamma(\alpha)} \int_{0}^{t}(t-s)^{\alpha-1} \mathrm{~d} s \\
& +\frac{M_{g}}{\Gamma(\alpha)} \int_{0}^{t}(t-s)^{\alpha-1}\left|z_{x}(s)-z_{y}(s)\right| \mathrm{d} s
\end{aligned}
$$

and

$$
\begin{aligned}
\left|z_{x}(t)-z_{y}(t)\right| & \leq\left|h\left(t, x(t), z_{x}(t)\right)-h\left(t, y(t), z_{y}(t)\right)\right| \\
& \leq K_{3}|x(t)-y(t)|+K_{4}\left|z_{x}(t)-z_{y}(t)\right| \\
& \leq \frac{K_{3}}{1-K_{4}}|x(t)-y(t)| .
\end{aligned}
$$

By replacing (8) in the inequality (7), we get

$$
\begin{aligned}
& |(P x)(t)-(P y)(t)| \leq \\
& \leq K_{1}|x(t)-y(t)|+K_{2}|\theta||x(t)-y(t)| \\
& \quad+K_{2}|x(t)-y(t)| \frac{M_{h}}{\Gamma(\alpha)} \int_{0}^{t}(t-s)^{\alpha-1} \mathrm{~d} s
\end{aligned}
$$




$$
\begin{aligned}
& +\frac{M_{g}}{\Gamma(\alpha)} \frac{K_{3}}{1-K_{4}} \int_{0}^{t}(t-s)^{\alpha-1}|x(s)-y(s)| \mathrm{d} s \\
\leq & K_{1}\|x-y\|+K_{2}\left(|\theta|+\frac{M_{h} t^{\alpha}}{\Gamma(\alpha+1)}\right)\|x-y\| \\
& +\frac{M_{g}}{\Gamma(\alpha)} \frac{K_{3}}{1-K_{4}}\left(\int_{0}^{t}(t-s)^{\alpha-1} \mathrm{~d} s\right)\|x-y\| \\
\leq & \left(K_{1}+K_{2}|\theta|+\left(M_{h} K_{2}+\frac{M_{g} K_{3}}{1-K_{4}}\right) \frac{t^{\alpha}}{\Gamma(\alpha+1)}\right)\|x-y\| \\
\leq & \left(K_{1}+K_{2}|\theta|+\left(M_{h} K_{2}+\frac{M_{g} K_{3}}{1-K_{4}}\right) \frac{T^{\alpha}}{\Gamma(\alpha+1)}\right)\|x-y\| .
\end{aligned}
$$

Then

$$
\|P x-P y\| \leq \beta\|x-y\| .
$$

By (6), the mapping $P$ is a contraction in $C([0, T], \mathbb{R})$. Hence $P$ has a unique fixed point $x \in C([0, T], \mathbb{R})$. Therefore $(1)$ has a unique solution.

Theorem 3. Assume that $f:[0, T] \times \mathbb{R} \rightarrow \mathbb{R}, g:[0, T] \times \mathbb{R} \rightarrow \mathbb{R} \backslash\{0\}$ and $h:[0, T] \times \mathbb{R} \times \mathbb{R} \rightarrow \mathbb{R}$ satisfy (H1)-(H3) and (6) holds. Then (1) has a unique solution $x$ and

$$
\begin{aligned}
|x(t)| \leq & \left(\frac{\left(1-K_{4}\right)\left(1-\left(K_{1}+K_{2}|\theta|\right)\right) \Gamma(\alpha+1)+M_{g} K_{3} K T^{\alpha}}{\left(1-K_{4}\right)\left(1-\left(K_{1}+K_{2}|\theta|\right)\right)^{2} \Gamma(\alpha+1)}\right) \\
& \times\left(Q_{1}+|\theta| Q_{2}+\frac{M_{g} Q_{3} T^{\alpha}}{\left(1-K_{4}\right) \Gamma(\alpha+1)}\right),
\end{aligned}
$$

where $Q_{1}=\sup _{t \in[0, T]}|f(t, 0)|, Q_{2}=\sup _{t \in[0, T]}|g(t, 0)|, Q_{3}=\sup _{t \in[0, T]}|h(t, 0,0)|$ and $K \in \mathbb{R}^{+}$is a constant.

Proof. Theorem 2 shows that the problem (1) has a unique solution. Let

$$
{ }^{C} D^{\alpha}\left(\frac{x(t)-f(t, x(t))}{g(t, x(t))}\right)=z_{x}(t), \quad x(0)=\theta g(0, x(0))+f(0, x(0)),
$$

then by Lemma $4, x(t)=f(t, x(t))+\theta g(t, x(t))+g(t, x(t)) I^{\alpha} z_{x}(t)$. Then by (H1), (H2) and (H3), for any $t \in[0, T]$ we have

$$
\begin{aligned}
|x(t)| \leq & |f(t, x(t))|+|\theta||g(t, x(t))|+|g(t, x(t))|\left|I^{\alpha} z_{x}(t)\right| \\
\leq & |f(t, x(t))-f(t, 0)|+|f(t, 0)| \\
& +|\theta|(|g(t, x(t))-g(t, 0)|+|g(t, 0)|)+M_{g}\left|I^{\alpha} z_{x}(t)\right| \\
\leq & K_{1}|x(t)|+Q_{1}+|\theta|\left(K_{2}|x(t)|+Q_{2}\right)+M_{g}\left|I^{\alpha} z_{x}(t)\right| .
\end{aligned}
$$

On the other hand, for any $t \in[0, T]$ we get

$$
\begin{aligned}
\left|z_{x}(t)\right| & =\left|h\left(t, x(t), z_{x}(t)\right)\right| \\
& \leq\left|h\left(t, x(t), z_{x}(t)\right)-h(t, 0,0)\right|+|h(t, 0,0)|
\end{aligned}
$$




$$
\begin{aligned}
& \leq K_{3}|x(t)|+K_{4}\left|z_{x}(t)\right|+|h(t, 0,0)| \\
& \leq \frac{K_{3}}{1-K_{4}}|x(t)|+\frac{Q_{3}}{1-K_{4}} .
\end{aligned}
$$

Therefore

$$
\begin{aligned}
|x(t)| \leq & K_{1}|x(t)|+Q_{1}+|\theta|\left(K_{2}|x(t)|+Q_{2}\right) \\
& +M_{g} I^{\alpha}\left(\frac{K_{3}}{1-K_{4}}|x(t)|+\frac{Q_{3}}{1-K_{4}}\right) .
\end{aligned}
$$

Thus

$$
\begin{aligned}
& \left(1-\left(K_{1}+K_{2}|\theta|\right)\right)|x(t)| \\
\leq & Q_{1}+|\theta| Q_{2}+\frac{M_{g} Q_{3} T^{\alpha}}{\left(1-K_{4}\right) \Gamma(\alpha+1)} \\
& +\left(\frac{M_{g} K_{3}}{\left(1-K_{4}\right)\left(1-\left(K_{1}+K_{2}|\theta|\right)\right)}\right)\left(I^{\alpha}\left\{\left(1-\left(K_{1}+K_{2}|\theta|\right)\right)|x(t)|\right\}\right) .
\end{aligned}
$$

By Lemma 3 , there is a constant $K=K(\alpha)$ such that

$$
\begin{aligned}
& \left(1-\left(K_{1}+K_{2}|\theta|\right)\right)|x(t)| \\
\leq & Q_{1}+|\theta| Q_{2}+\frac{M_{g} Q_{3} T^{\alpha}}{\left(1-K_{4}\right) \Gamma(\alpha+1)} \\
& +\left(\frac{M_{g} K_{3} K T^{\alpha}}{\left(1-K_{4}\right)\left(1-\left(K_{1}+K_{2}|\theta|\right)\right) \Gamma(\alpha+1)}\right) \\
& \times\left(Q_{1}+|\theta| Q_{2}+\frac{M_{g} Q_{3} T^{\alpha}}{\left(1-K_{4}\right) \Gamma(\alpha+1)}\right) \\
\leq & \left(\frac{\left(1-K_{4}\right)\left(1-\left(K_{1}+K_{2}|\theta|\right)\right) \Gamma(\alpha+1)+M_{g} K_{3} K T^{\alpha}}{\left(1-K_{4}\right)\left(1-\left(K_{1}+K_{2}|\theta|\right)\right) \Gamma(\alpha+1)}\right) \\
& \times\left(Q_{1}+|\theta| Q_{2}+\frac{M_{g} Q_{3} T^{\alpha}}{\left(1-K_{4}\right) \Gamma(\alpha+1)}\right) .
\end{aligned}
$$

Hence

$$
\begin{aligned}
|x(t)| \leq & \left(\frac{\left(1-K_{4}\right)\left(1-\left(K_{1}+K_{2}|\theta|\right)\right) \Gamma(\alpha+1)+M_{g} K_{3} K T^{\alpha}}{\left(1-K_{4}\right)\left(1-\left(K_{1}+K_{2}|\theta|\right)\right)^{2} \Gamma(\alpha+1)}\right) \\
& \times\left(Q_{1}+|\theta| Q_{2}+\frac{M_{g} Q_{3} T^{\alpha}}{\left(1-K_{4}\right) \Gamma(\alpha+1)}\right) .
\end{aligned}
$$

This completes the proof.

\section{Ulam Stability}

In this section, we study two types of Ulam stability of the hybrid implicit Caputo fractional differential equation (1) which are Ulam-Hyers and UlamHyers-Rassias stabilities. 
Lemma 5. Assume that $g$ satisfies (H1). If $y \in C([0, T], \mathbb{R})$ is a solution of the fractional differential inequality for each $\epsilon>0$

(9)

$$
\left|{ }^{C} D^{\alpha}\left(\frac{y(t)-f(t, y(t))}{g(t, y(t))}\right)-h\left(t, y(t),{ }^{C} D^{\alpha}\left(\frac{y(t)-f(t, y(t))}{g(t, y(t))}\right)\right)\right| \leq \epsilon,
$$

then, $y$ is a solution of the following inequality

$$
|y(t)-(P y)(t)| \leq \frac{\epsilon M_{g} T^{\alpha}}{\Gamma(\alpha+1)} .
$$

Proof. Let $y \in C([0, T], \mathbb{R})$ be a solution of the inequality (9) for each $\epsilon>0$. Then, from Remark 1 and Lemma 4 for some continuous function $\Psi(t)$ such that $|\Psi(t)|<\epsilon, t \in[0, T]$, we have

$$
\begin{aligned}
y(t)= & f(t, y(t))+\theta g(t, y(t))+\frac{g(t, y(t))}{\Gamma(\alpha)} \int_{0}^{t}(t-s)^{\alpha-1} \\
& \times\left(h\left(s, y(s),{ }^{C} D^{\alpha}\left(\frac{y(s)-f(s, y(s))}{g(s, y(s))}\right)\right)+\Psi(s)\right) \mathrm{d} s .
\end{aligned}
$$

Then, by Remark 1 and (H1), we obtain

$$
\begin{aligned}
|y(t)-(P y)(t)| & =\left|\frac{g(t, y(t))}{\Gamma(\alpha)} \int_{0}^{t}(t-s)^{\alpha-1} \Psi(s) \mathrm{d} s\right| \\
& \leq \frac{|g(t, y(t))|}{\Gamma(\alpha)} \int_{0}^{t}(t-s)^{\alpha-1}|\Psi(s)| \mathrm{d} s \\
& \leq \frac{\epsilon M_{g} T^{\alpha}}{\Gamma(\alpha+1)} .
\end{aligned}
$$

which is satisfied inequality (10). This completes the proof.

Theorem 4. Assume that the assumptions (H1)-(H3) are fulfilled and (6) holds. Then the problem (1) is Ulam-Hyers stable.

Proof. Under (H1)-(H3) and (6), the problem (1) has a unique solution in $C([0, T], \mathbb{R})$. Let $y \in C([0, T], \mathbb{R})$ be a solution of the inequality (9), then for each $t \in[0, T]$, we have

$$
\begin{aligned}
& |y(t)-x(t)| \\
& =\mid y(t)-f(t, x(t))+\theta g(t, x(t)) \\
& +\frac{g(t, x(t))}{\Gamma(\alpha)} \int_{0}^{t}(t-s)^{\alpha-1} h\left(s, x(s),{ }^{C} D^{\alpha}\left(\frac{x(s)-f(s, x(s))}{g(s, x(s))}\right)\right) \mathrm{d} s \mid \\
& =|y(t)-P y(t)+P y(t)-P x(t)| \\
& \leq|y(t)-P y(t)|+|P y(t)-P x(t)| \\
& \leq \frac{\epsilon M_{g} T^{\alpha}}{\Gamma(\alpha+1)}+\beta\|y-x\| \text {. }
\end{aligned}
$$


Then

By setting

$$
\|y-x\| \leq \frac{\epsilon M_{g} T^{\alpha}}{(1-\beta) \Gamma(\alpha+1)} .
$$

we obtain

$$
k=\frac{M_{g} T^{\alpha}}{(1-\beta) \Gamma(\alpha+1)},
$$

$$
|y(t)-x(t)| \leq k \epsilon
$$

Therefore, the problem (1) is Ulam-Hyers stable.

This completes the proof.

In the next, we introduce the following function.

(H4) The function $\phi \in C\left([0, T], \mathbb{R}^{+}\right)$is increasing and there exists a constant $\lambda_{\phi}>0$ such that, for each $t \in[0, T]$, we have

$$
I^{\alpha} \phi(t) \leq \lambda_{\phi} \phi(t) .
$$

Lemma 6. Assume that (H1) and (H4) are satisfied. If $y \in C([0, T], \mathbb{R})$ is a solution of the fractional differential inequality for each $\epsilon>0$

$$
\left|{ }^{C} D^{\alpha}\left(\frac{y(t)-f(t, y(t))}{g(t, y(t))}\right)-h\left(t, y(t),{ }^{C} D^{\alpha}\left(\frac{y(t)-f(t, y(t))}{g(t, y(t))}\right)\right)\right| \leq \epsilon \phi(t),
$$

then, $y$ is a solution of the following inequality

$$
|y(t)-(P y)(t)| \leq \epsilon M_{g} \lambda_{\phi} \phi(t) .
$$

Proof. Let $y \in C([0, T], \mathbb{R})$ be a solution of the inequality (11) for each $\epsilon>0$. Then, from Remark 1 and Lemma 4 for some continuous function $\Psi(t)$ such that $|\Psi(t)|<\epsilon \phi(t), t \in[0, T]$, we have

$$
\begin{aligned}
|y(t)-(P y)(t)| & =\left|\frac{g(t, y(t))}{\Gamma(\alpha)} \int_{0}^{t}(t-s)^{\alpha-1} \Psi(s) \mathrm{d} s\right| \\
& \leq \frac{|g(t, y(t))|}{\Gamma(\alpha)} \int_{0}^{t}(t-s)^{\alpha-1}|\Psi(s)| \mathrm{d} s \\
& \leq \frac{\epsilon M_{g}}{\Gamma(\alpha)} \int_{0}^{t}(t-s)^{\alpha-1} \phi(s) \mathrm{d} s \\
& \leq \epsilon M_{g} \lambda_{\phi} \phi(t) .
\end{aligned}
$$

which is satisfied inequality (12). This completes the proof.

Theorem 5. Assume that the assumptions (H1)-(H4) are fulfilled and (6) holds. Then the problem (1) is Ulam-Hyers-Rassias stable.

Proof. Under (H1)-(H4) and (6), the problem has a unique solution in $C([0, T], \mathbb{R})$. Let $y \in C([0, T], \mathbb{R})$ be a solution of the inequality (11), then for each $t \in[0, T]$, we have

$$
|y(t)-x(t)|
$$




$$
\begin{aligned}
= & \mid y(t)-f(t, x(t))+\theta g(t, x(t))+\frac{g(t, x(t))}{\Gamma(\alpha)} \int_{0}^{t}(t-s)^{\alpha-1} \\
& \times h\left(s, x(s),{ }^{C} D^{\alpha}\left(\frac{x(s)-f(s, x(s))}{g(s, x(s))}\right)\right) \mathrm{d} s \mid \\
= & |y(t)-(P y)(t)+(P y)(t)-(P x)(t)| \\
\leq & |y(t)-(P y)(t)|+|(P y)(t)-(P x)(t)| \\
\leq & \epsilon M_{g} \lambda_{\phi} \phi(t)+\beta\|y-x\| .
\end{aligned}
$$

Then

$$
|y(t)-x(t)| \leq \frac{\epsilon M_{g} \lambda_{\phi} \phi(t)}{1-\beta} .
$$

By taking a constant

$$
k_{\phi, f}=\frac{M_{g} \lambda_{\phi}}{1-\beta}
$$

we obtain

$$
|y(t)-x(t)| \leq k_{\phi, f} \epsilon \phi(t) .
$$

Therefore, the problem (1) is Ulam-Hyers-Rassias stable. This completes the proof.

\section{EXAMPLE}

Consider the following nonlinear problem

$$
\left\{\begin{array}{l}
{ }^{C} D^{\frac{3}{4}}\left(\frac{x(t)-\frac{1}{4} t \sin (x(t))}{1+\frac{1}{10} \exp \left(-t^{2}\right) \cos (x(t))}\right) \\
=\frac{1}{5} t \cos (x(t))+\frac{1}{5+\left|{ }^{C} D^{\frac{3}{4}}\left(\frac{x(t)-\frac{1}{4} t \sin (x(t))}{1+\frac{1}{10} \exp \left(-t^{2}\right) \cos (x(t))}\right)\right|}, t \in[0,1], \\
x(0)=g(0, x(0))+f(0, x(0)) .
\end{array}\right.
$$

where $\theta=1, f(t, x(t))=\frac{1}{4} t \sin (x(t)), g(t, x(t))=1+\frac{1}{10} \exp \left(-t^{2}\right) \cos (x(t))$ and

$$
\begin{aligned}
& h\left(t, x(t),{ }^{C} D^{\alpha}\left(\frac{x(t)-f(t, x(t))}{g(t, x(t))}\right)\right) \\
& =\frac{1}{5} t \cos (x(t))+\frac{1}{5+\left|{ }^{C} D^{\frac{3}{4}}\left(\frac{x(t)-\frac{1}{4} t \sin (x(t))}{1+\frac{1}{10} \exp \left(-t^{2}\right) \cos (x(t))}\right)\right|} .
\end{aligned}
$$

Set $h(t, u, v)=\frac{1}{5} t \cos (u)+\frac{1}{5+|v|}$. For any $u, v, u^{*}, v^{*} \in \mathbb{R}$ and $t \in[0,1]$, we have

$$
\begin{aligned}
\left|f(t, u)-f\left(t, u^{*}\right)\right| & \leq \frac{1}{4}\left|u-u^{*}\right| \\
\left|g(t, u)-g\left(t, u^{*}\right)\right| & \leq \frac{1}{10}\left|u-u^{*}\right| \\
\left|h(t, u, v)-h\left(t, u^{*}, v^{*}\right)\right| & \leq \frac{1}{5}\left|u-u^{*}\right|+\frac{1}{25}\left|v-v^{*}\right|,
\end{aligned}
$$


and

$$
|g(t, u)| \leq \frac{11}{10},|h(t, u, v)| \leq \frac{2}{5} .
$$

Hence, all conditions of Theorem 2 are fulfilled and $\beta=0.6393<1$, with

$$
M_{g}=\frac{11}{10}, M_{h}=\frac{2}{5}, K_{1}=\frac{1}{4}, K_{2}=\frac{1}{10}, K_{3}=\frac{1}{5}, K_{4}=\frac{1}{25} .
$$

Then the problem $(13)$ has a unique solution $x \in C([0, T], \mathbb{R})$. And from Theorem 4 we deduce that (13) is Ulam-Hyers stable.

Now, we choose $\phi(t)=t^{2}$ and in view of (2), we have

$$
I^{\alpha} \phi(t)=\frac{\Gamma(3)}{\Gamma(3+\alpha)} t^{2+\alpha} \leq \frac{2}{\Gamma\left(\frac{7}{2}\right)} t^{2}=\lambda_{\phi} \phi(t) .
$$

Thus condition (H4) is satisfied with $\phi(t)=t^{2}$ and $\lambda_{\phi}=\frac{2}{\Gamma\left(\frac{7}{2}\right)}=\frac{16}{15 \sqrt{\pi}}$, it follows from Theorem 5 that the problem (13) is Ulam-Hyers-Rassias stable. Acknowledgements. The authors are grateful to the referees for their valuable comments which have led to improvement of the presentation.

\section{REFERENCES}

[1] S. Abbas, Existence of solutions to fractional order ordinary and delay differential equations and applications, Electronic Journal of Differential Equations, 2011(9) (2011), 1-11.

[2] K. Aissani, M. Benchohra, Impulsive fractional differential inclusions with statedependent delay, Mathematica Moravica, 23(2) (2019), 97-113.

[3] R. P. Agarwal, Y. Zhou, Y. He, Existence of fractional functional differential equations, Computers and Mathematics with Applications, 59 (2010), 1095-1100.

[4] B. Ahmad, S. K. Ntouyas, Initial-value problems for hybrid Hadamard fractional differential equations, Electron. J. Differential Equations, 2014(161) (2014), 1-8.

[5] B. Ahmad, S. K. Ntouyas, Existence and uniqueness of solutions for CaputoHadamard sequential fractional order neutral functional differential equations, Electronic Journal of Differential Equations, 2017(36) (2017), 1-11.

[6] A. Ardjouni, Positive solutions for nonlinear Hadamard fractional differential equations with integral boundary conditions, AIMS Mathematics, 4(4) (2019), 1101-1113.

[7] A. Ardjouni, A. Djoudi, Positive solutions for first-order nonlinear Caputo-Hadamard fractional relaxation differential equations, Kragujevac Journal of Mathematics, 45(6) (2021), 897-908.

[8] A. Ardjouni, A. Djoudi, Existence of positive periodic solutions for third-order nonlinear delay differential equations with variable coefficients, Mathematica Moravica, 23(2) (2019), 17-28.

[9] A. Ardjouni, A. Djoudi, Approximating solutions of nonlinear hybrid Caputo fractional integro-differential equations via Dhage iteration principle, Ural Mathematical Journal, 5(1) (2019), 3-12.

[10] A. Ardjouni, A. Djoudi, Initial-value problems for nonlinear hybrid implicit Caputo fractional differential equations, Malaya Journal of Matematik, 7 (2019), 314-317.

[11] A. Ardjouni, A. Djoudi, Stability for nonlinear neutral integro-differential equations with variable delay, Mathematica Moravica, 19(2) (2015), 1-18.

[12] A. Ardjouni, A. Djoudi, Stability in nonlinear neutral differential equations with infinite delay, Mathematica Moravica, 18(2) (2014), 91-103. 
[13] S. Asawasamrit, W. Nithiarayaphaks, S. K. Ntouyas, J. Tariboon, Existence and stability analysis for fractional differential equations with mixed nonlocal conditions, Mathematics 7(117) (2019), 1-11.

[14] M. Benchohra, J. E. Lazreg, On stability for nonlinear implicit fractional differential equations, Le Matematiche 70(2) (2015), 49-61.

[15] M. Bohner, H. A. El-Morshedy, S. R. Grace, I. Sager, Oscillation of second-order nonlinear difference equations with sublinear neutral term, Mathematica Moravica, 23(1) (2019), 1-10.

[16] H. Boulares, A. Ardjouni, Y. Laskri, Positive solutions for nonlinear fractional differential equations, Positivity, 21 (2017), 1201-1212.

[17] H. Boulares, A. Ardjouni, Y. Laskri, Stability in delay nonlinear fractional differential equations, Rend. Circ. Mat. Palermo, 65 (2016), 243-253.

[18] A. Chidouh, A. Guezane-Lakoud, R. Bebbouchi, Positive solutions of the fractional relaxation equation using lower and upper solutions, Vietnam Jorunal of Mathematics, 44(4) (2016), 739-748.

[19] K. Diethelm, The Analysis of Fractional Differential Equations, Lecture Notes in Mathematics, Springer-Verlag, Berlin, Heidelberg, 2010.

[20] B. C. Dhage, Hybrid fixed point theory in partially ordered normed linear spaces and applications to fractional integral equations, Differential Equations \& Applications Ele-Math, 5 (2013), 155-184.

[21] B. C. Dhage, V. Lakshmikantham, Basic results on hybrid differential equations, Nonlinear Analysis-Hybrid Systems, 4 (2010), 414-424.

[22] B. C. Dhage, S. B. Dhage, S. K. Ntouyas, Approximating solutions of nonlinear hybrid differential equations, Applied Mathematics Letters, 34 (2014), 76-80.

[23] H. Gabsi, A. Ardjouni, A. Djoudi, Positive periodic solutions of second-order nonlinear neutral differential equations with variable coefficients, Mathematica Moravica, 22(2) (2018), 69-82.

[24] F. Ge, C. Kou, Stability analysis by Krasnoselskii's fixed point theorem for nonlinear fractional differential equations, Applied Mathematics and Computation, 257 (2015), 308-316.

[25] F. Ge, C. Kou, Asymptotic stability of solutions of nonlinear fractional differential equations of order $1<\alpha<2$, Journal of Shanghai Normal University, 44(3) (2015), 284-290.

[26] A. Guezane Lakoud, R. Khaldi, A. Kılıçman, Existence of solutions for a mixed fractional boundary value problem, Advances in Difference Equations, 2017(164) (2017), $1-9$.

[27] A. Guezane-Lakoud, S. Ramdane, Existence of solutions for a system of mixed fractional differential equations, Journal of Taibah University for Science, 12(4) (2018), 421-426.

[28] M. Haoues, A. Ardjouni, A. Djoudi, Existence, interval of existence and uniqueness of solutions for nonlinear implicit Caputo fractional differential equations, Transylvanian Journal of Mathematics and Mechanics, 10(1) (2018), 09-13

[29] D. Henry, Geometric Theory of Semi Linear Parabolic Equations, Springer -Verlag, Berlin, Heidelberge, New York, 1981.

[30] A. A. Kilbas, H. M. Srivastava, J. J. Trujillo, Theory and Applications of Fractional Differential Equations, Elsevier Science B. V., Amsterdam, 2006.

[31] C. Kou, H. Zhou, Y. Yan, Existence of solutions of initial value problems for nonlinear fractional differential equations on the half-axis, Nonlinear Analysis, 74 (2011), 59755986 . 
[32] K. D. Kucche, S. T. Sutar, On existence and stability results for nonlinear fractional delay differential equations, Boletim da Sociedade Paranaense de Matemática, 36(4) (2018), 55-75.

[33] V. Lakshmikantham, A. S. Vatsala, Basic theory of fractional differential equations, Nonlinear Analysis, 69 (2008), 2677-2682.

[34] N. Li, C. Wang, New existence results of positive solution for a class of nonlinear fractional differential equations, Acta Mathematica Scientia, 33 (2013), 847-854.

[35] I. Podlubny, Fractional Differential Equations, Academic Press, San Diego, 1999.

[36] I. A. Rus, Ulam stabilities of ordinary differential equations in a Banach space, Carpathian Journal of Mathematics, 26 (2010), 103-107.

[37] D. R. Smart, Fixed Point Theorems, Cambridge Tracts in Mathematics, 66, Cambridge University Press, London-New York, 1974.

[38] S. Zhang, The existence of a positive solution for a nonlinear fractional differential equation, Journal of Mathematical Analysis and Applications, 252 (2000), 804-812.

[39] J. Wang, L. Lv, Y. Zhou, Ulam stability and data dependence for fractional differential equations with Caputo derivative, Electronic Journal of Qualitative Theory of Differential Equations, 2011(63) (2011), 1-10.

[40] J. Wang, L. Lv, Y. Zhou, New concepts and results in stability of fractional differential equations, Communications in Nonlinear Science and Numerical Simulation, 17 (2012), 2530-2538.

Adel Lachouri

Applied Mathematics Lab

Department of Mathematics

UNIVERSITY OF ANNABA

P.O. Box 12, ANNABA

Algeria

E-mail address: lachouri.adel@yahoo.fr

Abdelouaheb Ardjouni

Department of Mathematics and Informatics

University of Souk Ahras

P.O. Box 1553, Souk Ahras

Algeria

E-mail address: abd_ardjouni@yahoo.fr

\author{
Ahcene Duoudi \\ Applied Mathematics Lab \\ Department of Mathematics \\ UnIVERSiTy OF ANNABA \\ P.O. Box 12, AnNABA \\ Algeria \\ E-mail address: adjoudi@yahoo.com
}

